\title{
Correction to: Development of a new phantom simulating extracellular space of tumor cell growth and cell edema for diffusion-weighted magnetic resonance imaging
}

\author{
Ryoji Mikayama ${ }^{1}$ Hidetake Yabuuchi ${ }^{2}\left(\right.$ He $\cdot$ Ryoji Matsumoto $^{3} \cdot$ Koji Kobayashi $^{3} \cdot$ Yasuo Yamashita $^{3}$. \\ Mitsuhiro Kimura ${ }^{1} \cdot$ Takeshi Kamitani $^{4} \cdot$ Koji Sagiyama $^{4} \cdot$ Yuzo Yamasaki $^{4}$
}

Published online: 14 February 2020

(c) European Society for Magnetic Resonance in Medicine and Biology (ESMRMB) 2020

\section{Correction to: \\ Magnetic Resonance Materials in Physics, Biology and Medicine \\ https://doi.org/10.1007/s10334-019-00823-6}

The original version of this article unfortunately contained a mistake. In Table 1, second column of "Cell edema" should read as:

$5 \%(10 \mu \mathrm{m})$

$17 \%(15 \mu \mathrm{m})$

$40 \%(20 \mu \mathrm{m})$

The corrected Table 1 is placed in the next page.
The original article can be found online at https://doi.org/10.1007/ s10334-019-00823-6.

Hidetake Yabuuchi

h-yabu@med.kyushu-u.ac.jp

Ryoji Mikayama

mikayama@r-tec.med.kyushu-u.ac.jp

Ryoji Matsumoto

r-matsu@ r-tec.med.kyushu-u.ac.jp

Koji Kobayashi

kokoba@med.kyushu-u.ac.jp

Yasuo Yamashita

yasuo-y@med.kyushu-u.ac.jp

Mitsuhiro Kimura

mitsuhirokimura0426@gmail.com

Takeshi Kamitani

kamitani@radiol.med.kyushu-u.ac.jp

Koji Sagiyama

sagiyama@ radiol.med.kyushu-u.ac.jp
Yuzo Yamasaki

yyama@ radiol.med.kyushu-u.ac.jp

1 Department of Health Sciences, Graduate School of Medical Sciences, Kyushu University, 3-1-1, Maidashi, Higashi-ku, Fukuoka, Fukuoka 812-8582, Japan

2 Department of Health Sciences, Faculty of Medical Sciences, Kyushu University, 3-1-1, Maidashi, Higashi-ku, Fukuoka, Fukuoka 812-8582, Japan

3 Division of Radiological Technology, Department of Medical Technology, Kyushu University Hospital, 3-1-1, Maidashi, Higashi-ku, Fukuoka, Fukuoka 812-8582, Japan

4 Department of Clinical Radiology, Graduate School of Medical Sciences, Kyushu University, 3-1-1, Maidashi, Higashi-ku, Fukuoka, Fukuoka 812-8582, Japan 
Table 1 The CV of the ADC value in tumor cell growth model and cell edema model for 3 months

\begin{tabular}{lclllll}
\hline Model & Particle ratio (diameter) & 0 month & 1 month & 2 months & 3 months & CV (\%) \\
\hline Tumor cell growth & $5 \%(10 \mu \mathrm{m})$ & $1.42 \pm 0.03$ & $1.36 \pm 0.03$ & $1.36 \pm 0.04$ & $1.36 \pm 0.03$ & 2.18 \\
& $20 \%(10 \mu \mathrm{m})$ & $1.30 \pm 0.04$ & $1.24 \pm 0.03$ & $1.23 \pm 0.04$ & $1.26 \pm 0.05$ & 2.46 \\
& $35 \%(10 \mu \mathrm{m})$ & $1.12 \pm 0.03$ & $1.09 \pm 0.03$ & $1.10 \pm 0.04$ & $1.09 \pm 0.05$ & 1.29 \\
& $50 \%(10 \mu \mathrm{m})$ & $0.89 \pm 0.05$ & $0.91 \pm 0.07$ & $0.96 \pm 0.06$ & $0.95 \pm 0.06$ & 3.56 \\
Cell edema & $5 \%(10 \mu \mathrm{m})$ & $1.42 \pm 0.03$ & $1.36 \pm 0.03$ & $1.36 \pm 0.04$ & $1.36 \pm 0.03$ & 2.18 \\
& $17 \%(15 \mu \mathrm{m})$ & $1.30 \pm 0.03$ & $1.25 \pm 0.02$ & $1.25 \pm 0.04$ & $1.26 \pm 0.04$ & 1.89 \\
& $40 \%(20 \mu \mathrm{m})$ & $1.09 \pm 0.03$ & $1.09 \pm 0.03$ & $1.07 \pm 0.03$ & $1.08 \pm 0.06$ & 0.88 \\
\hline
\end{tabular}

Data are means \pm standard deviations

$C V$ coefficient of variation

Publisher's Note Springer Nature remains neutral with regard to jurisdictional claims in published maps and institutional affiliations. 